\title{
PRIME KNOTS AND TANGLES
}

BY

\author{
W. B. RAYMOND LICKORISH
}

\begin{abstract}
A study is made of a method of proving that a classical knot or link is prime. The method consists of identifying together the boundaries of two prime tangles. Examples and ways of constructing prime tangles are explored.
\end{abstract}

Introduction. This paper explores the idea of the prime tangle that was briefly introduced in $[\mathbf{K L}]$. It is here shown that summing together two prime tangles always produces a prime knot or link. Here a tangle is just two arcs spanning a 3-ball, and such a tangle is prime if it contains no knotted ball pair and its arcs cannot be separated by a disc. A few prototype examples of prime tangles are given (Figure 2), together with ways of using them to create infinitely many more (Theorem 3). Then, usage of the prime tangle idea becomes a powerful machine, nicely complementing other methods, in the production of prime knots and links. Finally it is shown (Theorem 5) that the idea of the prime tangle has a very natural interpretation in terms of double branched covers.

The paper should be interpreted as being in either the P.L. or smooth category. With the exception of the section on double branched covers, all the methods used are the straightforward (innermost disc) techniques of the elementary theory of 3-manifolds. None of the proofs is difficult; the paper aims for significance rather than sophistication. Indeed various possible generalizations of the prime tangle have not been developed (e.g. $n$ arcs, for $n>2$, or arcs in a ball-with-holes meeting each boundary component in four points) in order to avoid unnecessary complication.

The author wishes to record his gratitude to the University of California at Santa Barbara for providing opportunity, facilities and inspiration for the writing of this paper.

\section{Sums of prime tangles.}

Definition. A (2-string) tangle is a pair $(B, t)$ where $B$ is a 3-ball and $t$ is a pair of disjoint arcs in $B$ with $t \cap \partial B=\partial t$. (Examples appear in Figure 2.) Two tangles, $\left(B_{1}, t_{1}\right)$ and $\left(B_{2}, t_{2}\right)$, are equivalent if there is a homeomorphism of pairs from $\left(B_{1}, t_{1}\right)$ to $\left(B_{2}, t_{2}\right)$. A tangle $(B, t)$ is untangled if it is equivalent to $(D \times I,\{x, y\}$ $\times I)$, where $D$ is a disc containing points $x$ and $y$ in its interior.

This definition may be deceptively simple. In particular no orientations have been placed on the 3-balls nor on the arcs they contain. Further, in the definition

Received by the editors August 26, 1980.

1980 Mathematics Subject Classification. Primary 57M25; Secondary 57M12, 57N10.

Key words and phrases. Prime knot, tangle, branched cover, irreducible 3-manifold. 
of tangle equivalence no requirement has been made that the homeomorphism be, in any sense, the identity on the boundary of the ball (so this usage differs slightly from $[\mathbf{C}])$. In particular Figure 1 is $a$ picture of an untangle.

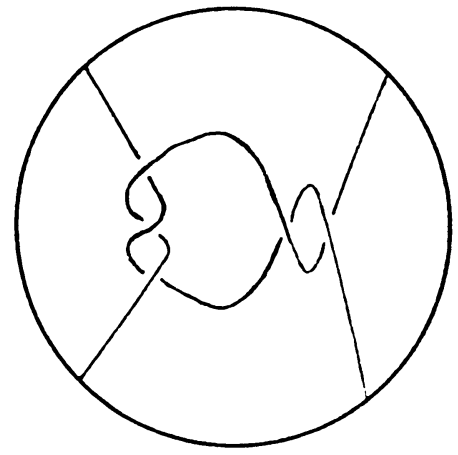

FIGURE 1

In [KL] the definition of a prime tangle was given as follows.

Definition. A tangle $(B, t)$ is prime if it has the following properties:

(1) Any 2-sphere in $B$, which meets $t$ transversely in two points, bounds in $B$ a ball meeting $t$ in an unknotted spanning arc;

(2) $(B, t)$ is not untangled.

Note that condition (2) is, in the presence of (1), equivalent to the condition that there is no disc properly embedded in $B$ which separates the two arcs of $t$. If there were such a disc it would, by (1), separate $B$ into two balls each containing an arc of $t$ as an unknotted spanning arc and $(B, t)$ would thus be untangled. It is hoped that tangles satisfying the definition will be regarded as deserving of the prestigious name 'prime', although certainly a case could be made for a more restrictive definition.

The interest in prime tangles focuses on their use in the creation and detection of prime knots and links. Recall that a link $l$ of disjoint simple closed curves in $S^{3}$ is prime if there is no $S^{2}$ in $S^{3}$ that separates the components of $l$, and any $S^{2}$ that meets $l$ in two points, transversely, bounds in $S^{3}$ one and only one ball intersecting $l$ in a single unknotted spanning arc. For links of one component this reduces to the usual definition of a prime knot; the unknot is not prime. If $\left(B_{1}, t_{1}\right)$ and $\left(B_{2}, t_{2}\right)$ are tangles and $f:\left(\partial B_{1}, \partial t_{1}\right) \rightarrow\left(\partial B_{2}, \partial t_{2}\right)$ is a homeomorphism, a link of one or two components in $S^{3}$ can be obtained by identifying the boundaries of the tangles using $f$. The result, $\left(B_{1}, t_{1}\right) \cup \cup_{f}\left(B_{2}, t_{2}\right)$ will be referred to as $a$ sum of the two tangles. Of course, different links result from different choices of $f$, and, essentially, choices of $f$ can range over the classical braid group of four strings in $S^{2} \times I$. The following theorem, which has a fairly elementary proof, asserts that any such sum of two prime tangles is a prime link. Thus, by varying $f$, a few simple examples of prime tangles produce a plethora of prime links.

THEOREM 1. Let $k$ be a link of one or two components in $S^{3}$. Suppose that $S^{2}$ is a 2-sphere in $S^{3}$ meeting $k$ transversely in four points and separating $S^{3}$ into 3-balls $A$ and $B$, so that $(A, A \cap k)$ and $(B, B \cap k)$ are both prime tangles. Then $k$ is a prime link. 
Proof. First note that $k$ cannot be the unknot: If $k$ bounds a disc $D$, which may be assumed to be transverse to $S^{2}, D \cap S^{2}$ is the disjoint union of two spanning arcs and a collection of simple closed curves. If $C$ is such a simple closed curve, innermost on $D$, let $C$ be the boundary of a disc $D^{\prime}$ in $D$. This $D^{\prime}$ is, without loss of generality, contained in $A$ and, by primeness, does not separate the arcs of $A \cap k$ in $A$. Thus, without moving $k, D^{\prime}$ can be isotopped off $A$ reducing the number of components of $D \cap S^{2}$. Hence it may be assumed that $D \cap S^{2}$ is just two arcs spanning $D$. This means that one of the two prime tangles is untangled and that is a contradiction.

Now suppose that $F$ is a 2 -sphere in $S^{3}$ meeting $k$ transversely in two points; it is required to show that, on one side, $F$ bounds a ball meeting $k$ in an unknotted arc. The definition of a prime tangle implies that this is true if $F$ is entirely contained in $A$ or in $B$, so it is in order to proceed by induction on the number of components of $F \cap S^{2}$. It may be assumed that $F$ intersects $S^{2}$ transversely in simple closed curves. Let $C$ be such a curve innermost on $F$ that bounds a disc $D$ in $F$; it may be assumed without loss of generality that $D$ is in $A$. This $C$ may be chosen so that $D \cap k=\varnothing$ or $D \cap k$ is a single point (for $F \cap k$ is just two points and some innermost disc will not contain them both). If $D \cap k$ is empty, as before, $D$ cannot separate the arcs of $A \cap k$ and therefore there is an isotopy, fixed on $k$, which reduces the number of components of $F \cap S^{2}$. If, on the other hand, $D \cap k$ is one point, then $C$ must bound a disc $D^{\prime}$ in $S^{2}$ which also meets $k$ in one point. Then, $D \cup D^{\prime}$ bounds a ball in $A$ which meets $k$ in an arc. Because $(A, A \cap k)$ is prime, this arc is unknotted and so there is an isotopy that reduces the number of components of $F \cap S^{2}$ and which keeps $k$ set-wise fixed. Hence, by the induction, $F$ bounds a ball meeting $k$ in an unknotted arc.

Finally, if $F$ is now a 2-sphere in $S^{3}$ disjoint from $k$, then by exactly the same argument as before, $F$ may be isotopped into $A$ keeping $k$ fixed. Thus $F$ bounds a ball disjoint from $k$, and thus $k$ cannot be a split link. Hence $k$ is a prime link.

2. Examples of prime tangles. A selection of tangles is depicted in Figure 2. That each of these tangles is prime can be shown as follows.

(a) If there were a 3-ball in this tangle meeting the arcs of the tangle in just one knotted arc, then such a knotted arc-ball pair would persist as a summand of any knot created by adding (in the sense of $\$ 1$ ) another tangle to the given one. However one can, in an obvious manner, add the untangle so as to create the unknot in which there is certainly no nontrivial summand. The given tangle cannot be the untangle as one of its arcs is a knotted spanning arc of the ball.

(b) This diagram represents a knotted spanning arc of the ball and another arc running parallel to it. The figure shows the arcs knotted in trefoil knots, but any knot will suffice. The proof that the tangle is prime is exactly the same as for tangle (a).

(c) This tangle contains no knotted arc-ball pair because each of the arcs of the tangle is unknotted (this remark applies equally well to tangles (d)-(g)). The tangle is not the untangle because any sum (in the sense of $\S 1$ ) of two untangles is a 2-bridge knot or link (a rational knot or link in the terminology of [C]). The untangle can be added to tangle (c) to produce the square knot and this is not a 
2-bridge knot. This last fact is shown directly in [Gk] by proving that the group of the square knot needs more than two generators for a presentation; it also follows from the theorem of $\mathrm{H}$. Schubert [S] that a 2-bridge knot is prime.

(d) and (e) These two tangles are prime for the same reasons as was tangle (c), the square knot being replaced in the argument by the granny knot for (d) and by the sum of a trefoil and a tweeny knot $\left(5_{2}\right)$ for the tangle (e).

(f) and (g) Neither of these contains a knotted arc-ball pair (see (c)). The untangle can be added to (f) (by joining outside the diagram the top left point on the boundary to the bottom right, and the top right to the bottom left) to produce the seven-crossing alternating link $7_{6}^{2}$ (or $\cdot 2$, see [C]). In exactly the same way the untangle can be added to $(\mathrm{g})$ to give the eight crossing knot $8_{17}$ (or $\left.\cdot 2 \cdot 2\right)$. Neither $7_{6}^{2}$ nor $8_{17}$ is 2-bridged, so tangles (f) and (g) are not untangled.

From these seven examples an infinity of prime knots can be constructed by adding them together in various ways (Theorem 1). It is interesting to note that the two eleven-crossing knots with trivial Alexander polynomial (namely .- $(2,3) .2$ and .- $(3,2) .2$., see $[\mathbf{C}])$ are both sums of tangle (a) with tangle (f). It can then be deduced immediately from Theorem 1 that these knots are both prime and hence knotted (the latter fact being not so trivial in the absence of any abelian knot invariants).

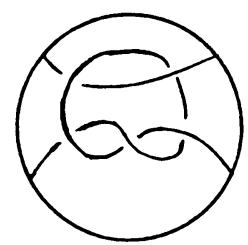

(a)

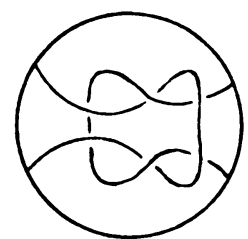

(c)

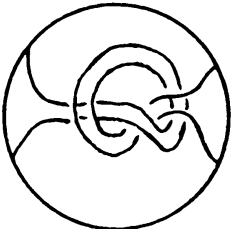

(b)

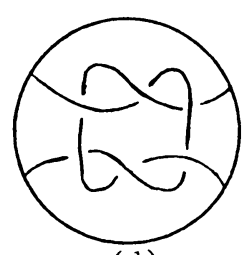

(d)

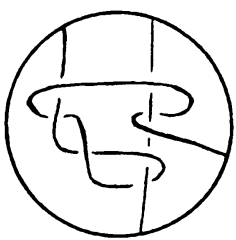

(e)

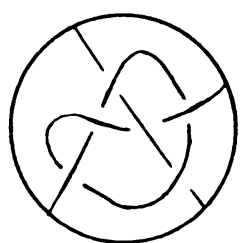

(f)

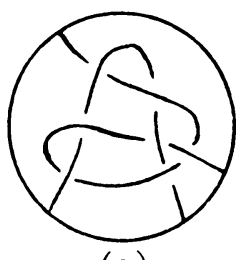

(g)

Figure 2

A little experimentation leads to the conclusion that any diagram of a prime tangle must have at least five crossovers. Although (for example $8_{17}$ ) a sum of two prime tangles might have a presentation with less than ten crossings this does not 
seem to be a very important question. It does, however, mean that diagrams of knots with less than ten crossings are not going to readily suggest ways of expressing those knots as prime tangle sums. Thus in the compilation of tables of prime knots Theorem 1 is not much use for low crossing numbers. It is however quite impressive to glance at diagrams of knots of eleven crossings and note that a large proportion of them can be proved to be prime using Theorem 1 and tangles (a)-(g). In [P2] K. A. Perko gives a list, complete with diagrams, of all known knots that appear to have crossing number eleven and that are prime (composite knots are traditionally omitted from knot tables). Perko outlines his methods of establishing primeness in [P1]. They rely heavily on Schubert's formula $[S]$ for the additivity of the bridge number, $b(k)$, of a knot, namely that $b\left(k_{1}+k_{2}\right)+1=$ $b\left(k_{1}\right)+b\left(k_{2}\right)$. Now, $b(k)=1$ if and only if $k$ is unknotted, so the formula immediately implies that a knot with bridge number equal to two (a rational knot [C]) is prime. Further, if $k$ is composite and $b(k)=3, k$ can only be a sum of knots with bridge number two. In [P1] it is shown that a large class of these knots with $b(k)=3$, namely the weakly 2 -bridge knots, are prime. It is interesting to note that of the 552 knots in Perko's list of 11 crossing knots, only 15 have $b(k)>3$ and so cannot have their primeness readily established by bridge number techniques. However, when $b(k)>3$, the knot $k$ can easily have achieved enough complexity to be a sum of two prime tangles. For example, each of the knots $11_{426}$ through $11_{434}$ is a sum of two of the tangles of Figure 2; hence each is prime.

The following lemma states that if any tangle is removed from the middle of a prime tangle and is replaced by another prime tangle, then the result is prime. In this way prime tangles of enormous complexity can be constructed; Figure 3 shows tangle (a) replacing the untangle in tangle (f).

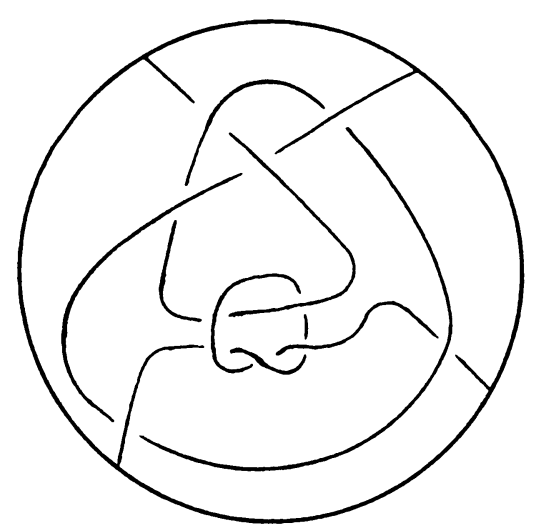

FIGURE 3

LeMmA 2. Let $(A, t)$ be a (two-string) tangle that is either prime or untangled. Let $B$ be a 3-ball in the interior of $A$ which meets each component of $t$ in a single interval. Let $u$ be two arcs in $B$ such that $(B, u)$ is a prime tangle and $\partial u=\partial B \cap t$. Let $t^{\prime}$ denote the two arcs $(t-(t \cap B)) \cup u$; then $\left(A, t^{\prime}\right)$ is a prime tangle. 
Proof. The proof is similar to that of Theorem 1. Suppose that $F$ is a 2-sphere contained in $A$, with $F \cap t^{\prime}$ a pair of points, the intersection being transverse. If $F$ is in $B$ then certainly $F$ bounds a ball meeting $t^{\prime}$ in an unknotted arc because $(B, u)$ is prime. If $F$ is disjoint from $B$ the same is true using the fact that $(A, t)$ is prime or untangled. Thus the proof proceeds by induction on the number of components of $F \cap \partial B$. Suppose that $C$ is a simple closed curve of $F \cap \partial B$, innermost on $F$,

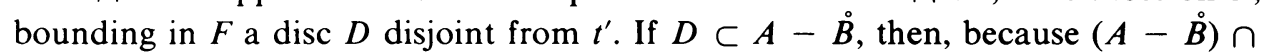
$t^{\prime}$ is four arcs each joining $\partial B$ to $\partial A, C$ also bounds a disc $D^{\prime}$ in $\partial B$ with $D^{\prime} \cap t^{\prime}$ being empty. Hence there is an ambient isotopy of $A$, fixed on $t^{\prime}$ (sliding $D$ over $\left.D^{\prime}\right)$ which reduces the number of components of $F \cap \partial B$. If $D \subset B, D$ cannot separate the arcs of $u$ as $(B, u)$ is prime, and thus once again the disc $D^{\prime}$ exists and there is an isotopy reducing the number of components of $F \cap \partial B$. If $D \cap t^{\prime}$ is a single point (note that $D$ may be chosen so that $D \cap t^{\prime}$ is never more than a single point), exactly as in the proof of Theorem 1 an isotopy may be constructed, keeping $t^{\prime}$ fixed set-wise, to reduce $F \cap \partial B$.

It remains to check that $\left(A, t^{\prime}\right)$ is not untangled. Suppose that $\Delta$ is a disc properly contained in $A$, separating the two components of $t^{\prime}$. Assume that $\Delta$ is transverse to $\partial B$, and isotop $\Delta$ using the above innermost disc argument until $\Delta \cap \partial B$ is empty. Now the whole of $B$ must lie in one of the components of $A-\Delta$ and this contradicts the fact that $B$ meets each of the components of $t^{\prime}$.

$A n$ obvious way of adding two tangles together is to identify a (disc, point pair) in the boundary of one tangle with a (disc, point pair) in the boundary of the other so that the result is still a tangle. The result will be called a partial sum of the two tangles; there are, of course, infinitely many partial sums of two given tangles. The following theorem shows that any partial sum of two prime tangles is a prime tangle.

Theorem 3. Let $(C, v)$ be a (two-string) tangle, and let $D$ be a disc properly contained in $C$ that separates $(C, v)$ into two tangles $(A, t)$ and $(B, u)$. If $(A, t)$ is prime or is untangled, $D$ meets both arcs of $(A, t)$, and $(B, u)$ is prime, then $(C, v)$ is prime.

Proof. Let $(\partial B, \partial u) \times I \subset(B, u)$ be a collar of $\partial B$ compatible with $u$. Let $(\hat{B}, \hat{u})$ be the closure of the complement of this collar in $(B, u)$. Then $(\hat{B}, \hat{u})$ is a subtangle of $(C, v)$ which can be replaced by the untangle to give a copy of $(A, t)$. Application of Lemma 2 to this copy of $(A, t)$ and the tangle $(\hat{B}, \hat{u})$ gives the result immediately.

Using the concepts of sums and partial sums of tangles it is easy to reprove the main result of $[\mathbf{K L}]$.

TheOREM 4. Any knot of $S^{1}$ in $S^{3}$ is concordant to a prime knot.

Proof. It will be shown below that any knot is a sum of a prime tangle with the untangle; but, the untangle is concordant to the prime tangle (c) of Figure 2, so the knot is concordant to a sum of two prime tangles, which is a prime knot by Theorem 1 . 
If $k_{1}$ is a prime knot, let $D$ be a disc meeting $k_{1}$ transversely in two points so that $\partial D$ is nul-homologous but not nul-homotopic in $S^{3}-k_{1}$ (for example, $\partial D$ can be taken to be a loop encircling a cross-over of some presentation of $\left.k_{1}\right)$. Let $\left(A_{1}, t_{1}\right)$ be the tangle consisting of $\left(S^{3}, k_{1}\right)$ less a neighbourhood of $D$. Let $F$ be a 2 -sphere in $A_{1}$, meeting $t_{1}$ transversely in two points. If $F$ bounds a knotted ball pair, the remainder of $\left(S^{3}, k_{1}\right)$ is, by the primeness of $k_{1}$, unknotted, and then $\partial D$ is nul-homotopic in $S^{3}-k_{1}$. Thus $\left(A_{1}, t_{1}\right)$ is either prime or untangled, and $\left(A_{1}, t_{1}\right)$ summed with an untangle produces $\left(S^{3}, k_{1}\right)$. However $a$ partial sum of $\left(A_{1}, t_{1}\right)$ with prime tangle (b), or (a), of Figure 2, produces another tangle $\left(\bar{A}_{1}, \bar{t}_{1}\right)$ which can also be summed with the untangle to give $\left(S^{3}, k_{1}\right)$. By Theorem $3,\left(\bar{A}_{1}, \bar{t}_{1}\right)$ is a prime tangle. If now $k$ is any knot, $k$ can be expressed as $\sum_{1}^{n} k_{i}$, a sum of prime knots. For each component a prime tangle $\left(\bar{A}_{i}, \bar{t}_{i}\right)$ can be constructed as above, and a partial sum of all the $\left(\bar{A}_{i}, \bar{t}_{i}\right)$ summed with the untangle as depicted in Figure 4 reproduces $\left(S^{3}, k\right)$.

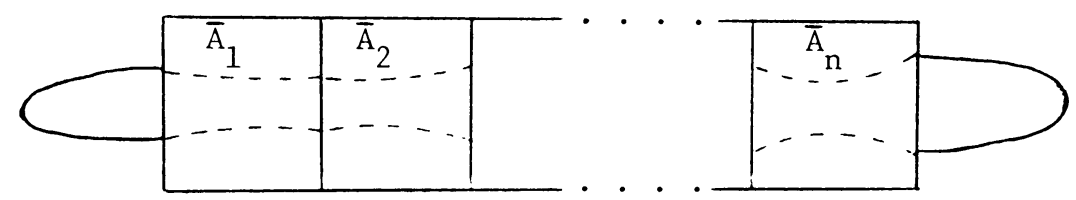

Figure 4

3. Double branched covers of tangles. The theory of knots and links has always been related to the study of covers of $S^{3}$ branched over knots or links. Thus it is pleasing that the idea of a prime tangle has a very natural interpretation in terms of double branched covers. The relevant result is the following.

THEOREM 5. A two-string tangle $(A, t)$ is prime if and only if the double cover of $A$ branched over (both components of) $t$ is both irreducible and boundary-irreducible.

Of course, the boundary of the double cover of $\partial A$ branched over $\partial t$ is the double cover of a sphere branched over four points, and this is a torus. A manifold with boundary a torus is irreducible and boundary-irreducible if every 2-sphere contained in it bounds a 3-ball, and it is not a solid torus. The proof of the theorem, given below, is elementary only because it relies heavily on the $\mathbf{Z}_{\mathbf{2}}$-versions of the loop and sphere theorems and on the $\mathbf{Z}_{2}$-version of the Smith Conjecture. This last result is due to $F$. Waldhausen [W], whilst the first two theorems are proved by P. K. Kim and J. L. Tollefson [KT].

Proof of Theorem 5. Suppose that $(A, t)$ is a prime tangle and let $p: M \rightarrow A$ be the double cover of $A$ branched over $t$. Now, $\mathbf{Z}_{2}$ acts on $M$ with generator $g$ as the group of covering translations; $g$ is an involution with two arcs as its fixed point-set. If $M$ is reducible there exists a 2 -sphere $S$ in $M$, not bounding a ball, which, by the $\mathbf{Z}_{2}$ sphere theorem [KT], may be chosen so that either $g S=S$ or $g S \cap S=\varnothing$. If $g S \cap S=\varnothing$ then $p S$ is a sphere embedded in $A-t$; thus $p S$ bounds a ball in $A-t$ which lifts to a ball in $M$ with $S$ as boundary, and this cannot exist. If $g S=S$, then $g \mid S$ is an involution with two fixed points, so $p S$ is a 
2-sphere in $A$ meeting $t$ in two points. By the primeness of $(A, t)$ it follows that $p S$ bounds a ball in $A$ meeting $t$ in an unknotted arc. The lift of this ball is then a ball with $S$ as boundary. This is again a contradiction and therefore $M$ is irreducible. It remains to check that $M$ is not a solid torus. Suppose $M$ is a solid torus. Then there is a disc $D$ properly embedded in $M$, with $\partial D$ essential in $\partial M$, such that either $g D=D$ or $g D \cap D=\varnothing$ (this uses the $\mathbf{Z}_{2}$ loop theorem [KT]). In the first case $p D$ is a disc in $A$ meeting $t$ in one point; then $\partial(p D)$ bounds a disc in $\partial A$ meeting $\partial t$ in one point, and that disc lifts to a disc in $\partial M$ with $\partial D$ as its boundary. Otherwise, $g D \cap D=\varnothing$ and $p D$ is an embedded disc with $p D \cap t=\varnothing$. As $p D$ cannot separate the arcs of $t, \partial(p D)$ bounds a disc in $\partial A-\partial t$, and this lifts to a disc in $M$ with $\partial D$ as its boundary. This is again a contradiction, so no such disc $D$ exists, and $M$ is not a solid torus.

Conversely, suppose that $p: M \rightarrow A$ is the double cover of a 3-ball $A$ branched over a tangle $t$, and that $M$ is irreducible and boundary-irreducible. Suppose that $B$ is a 3-ball in $A$ such that $B \cap t$ is a single arc. Then $p^{-1}(\partial B)$, being the double cover of a 2 -sphere branched over two points, is a 2-sphere in $M$. It follows from the irreducibility of $M$ that $p^{-1} B$ is a 3-ball. But the covering translation $g$ acts on the ball $p^{-1} B$ with the $\operatorname{arc} p^{-1}(B \cap t)$ as fixed point set. By the solution to the $\mathbf{Z}_{2}$ Smith Conjecture, this arc is unknotted and the action is conjugate to a standard rotation. Hence $(B, B \cap t)$ is an unknotted arc-ball pair. Finally, note that $(A, t)$ cannot be untangled because the double branched cover would then be a solid torus.

It is shown in [KT], using much the same methods as are used here for Theorem 5 , that a link $l$ in $S^{3}$ is prime if and only if the double cover of $S^{3}$ branched over $l$ is irreducible. Also, it is well known (and easy to prove) that if a 3-manifold $M$ is formed by identifying together the boundaries of two irreducible boundary-irreducible 3-manifolds, then $M$ itself is irreducible. These two facts together with Theorem 5 immediately produce an alternative proof of Theorem 1 . However the proof originally given in $\S 1$ was entirely elementary avoiding anything as sophisticated as a branched cover and its associated theory. This parallelism of proof should be compared with that existing in the proof that a knot with bridge number two is prime. One proof uses the fact that such a knot, being a sum of two untangles, has as its associated double branched cover of $S^{3}$ a lens space. A lens space is irreducible because its universal cover, $S^{3}$, is irreducible, so, by the above-quoted result, the knot is prime. The other proof has already been mentioned in $\$ 2$ and is an immediate consequence of Schubert's nontrivial result [S] on the additivity of $(b(k)-1)$.

From the perspective of this paper, the motivation of Theorem 5 is that it produces examples of irreducible, boundary-irreducible 3-manifolds from the infinity of prime tangles produced in $\$ 2$.

Two other methods of establishing the primeness of a knot are reasonably well known. The first uses the additivity of the genus of knots, so that knots with genus 1 are prime. The second, originated by Schubert, uses the fact that if a knot $k$ is contained in a knotted solid torus $T$ in $S^{3}$ with the property that there are no 
knotted arc-ball pairs in $(T, k)$, and any disc spanning $T$ meets $k$ in at least two points, then $k$ is prime. This too can be proved by simple innermost disc methods. Either of these two methods proves that the double of a knot is prime; the second method was used by Livingston [L] to give another proof of Theorem 4 . The interesting thing to note about the second method is that $\partial T$ is a torus, and that if $p: M \rightarrow S^{3}$ is the double cover of $S^{3}$ branched over $k$, then $p^{-1}(\partial T)$ is one or two tori which separate $M$ into pieces that are irreducible and boundary irreducible. Then $k$ is prime by [KT]. Thus this method and the main method of this paper consist of detecting incompressible tori in the double branched cover, and establishing that the tori separate the cover into irreducible components. The main results and examples of the paper are intended to show that in practice this can of ten be done by using the idea of the prime tangle.

When it is known that a knot is prime, then the double branched cover $M$ is irreducible, and $M$ can be analyzed by 3-manifold techniques nearly all of which require that the 3-manifold be irreducible. In particular the theory of the characteristic variety of K. Johannson [Jn] and of W. Jaco and P. Shalen [Jo] in its equivariant form due to $F$. Bonahon [B] can be used. This considers maximal collections of incompressible tori in $M$, no two being parallel, and deletes tori from such a collection, in order to minimize the number of ends of the components of the complement of these tori that have Seifert Fibre structures. (E.g. if a torus separates two components and the union of these components with the torus has a Seifert Fibre structure, that torus is removed from the collection.) These tori are unique up to isotopy and may be taken to be invariant under the covering translation, so they will correspond to a well-defined unique set of tori, in the complement of the original knot, and 2-spheres meeting the knot at four points. It is only in this sort of sense that one can hope to obtain a unique decomposition of the knot into prime "tangles". This situation has been well explored by L. C. Siebenmann and F. Bonahon. A simple example is shown in Figure 5, in which two

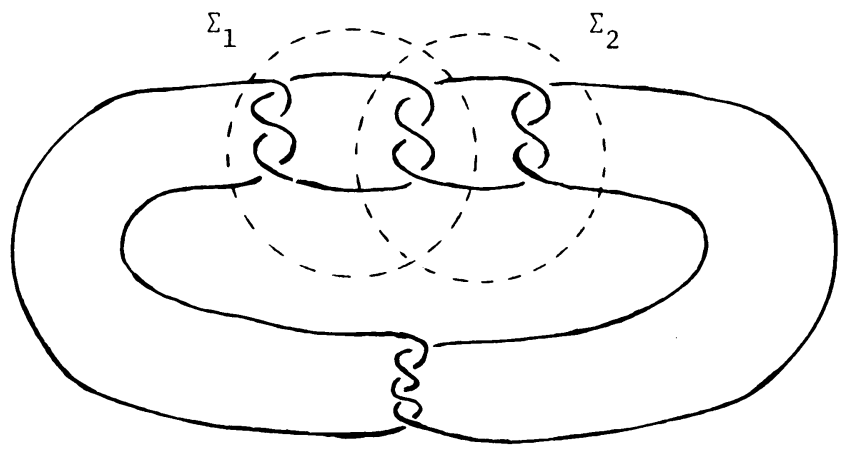

Figure 5

spheres $\Sigma_{1}$ and $\Sigma_{2}$ are indicated, each of which separates the knot $k$ into two prime tangles. This knot is a plumbing knot or an algebraic knot in the sense of Conway [C] (i.e., it is a sum of an untangle with a tangle formed by partially summing a finite collection of untangles together). The double cover of $S^{3}$ branched over $k$ is, 
in the given case, a Seifert Fibre space, the two spheres lift to incompressible tori, but of course neither torus appears in a set of tori (empty in this case) defining the characteristic variety. Hence there is no canonical way to break this knot up into a sum of prime tangles.

I he following proposition and its corollary do however give a somewhat limited result on the uniqueness of factorization of prime tangles by partial summation.

Proposition 6. Let $(A, t)$ be a tangle that is either prime or untangled. Let $\Delta$ be a disc properly embedded in $A$ that cuts each arc of $t$ in one point and separates $(A, t)$ into two tangles neither of which can be regarded as $(\Delta, \Delta \cap t) \times I$. Let $D$ be another disc with exactly the same properties. Then there exists an ambient isotopy of $A$, fixing $t$ setwise, that moves $D$ to a position in which $\Delta \cap D$ is a collection of nested simple closed curves in $\Delta$ encircling both points of $\Delta \cap t$. (Note that $D$ and $\Delta$ lift to essential annuli in the double branched cover.)

Corollary. In $\partial A-\partial t, \partial D$ is isotopic to $\partial \Delta$.

Proof. Consider the (transverse) intersections of $D$ with $\Delta$. Any simple closed curves of this intersection that encircle, in $\Delta$, no point of $\Delta \cap t$ may be removed by isotopy of $D$, beginning with those innermost on $\Delta$. Next, using the primeness of $(A, t)$ those simple closed curves that encircle one point of $\Delta \cap t$ may be removed in the same way. Now consider arcs of intersection of $D$ with $\Delta$. Suppose $\alpha$ is such an arc extreme-most on $\Delta$, so that there is a component $\Delta_{1}$ of $\Delta-\alpha$ disjoint from $D$. Now $\alpha$ separates $D$ into discs $D_{1}$ and $D_{2}$. There are various possibilities to consider. Suppose $\Delta_{1} \cap t=\varnothing$. If $D_{1} \cap t=\varnothing D_{1}$ can be slid across $\Delta_{1}$ reducing the number of components of $D \cap \Delta$; similarly if $D_{2} \cap t=\varnothing$. If $D_{1} \cap t$ and $D_{2} \cap t$ are each one point then, using the primeness of $(A, t), D$ separates from $(A, t)$ a trivial tangle consisting of two unknotted arc-ball pairs glued together along $\Delta_{1}$. This is a tangle of the type debarred by hypothesis. Hence it may be assumed that $\alpha$ separates the two points of $\Delta \cap t$, so now suppose $\Delta_{1} \cap t$ is one point.

By the symmetry between $\Delta$ and $D$ it may be assumed that $\alpha$ separates the two points of $D \cap t$, so that $D_{1} \cap t$ and $D_{2} \cap t$ are each one point. Because $D$ meets both arcs of $t$, one of these points, $D_{1} \cap t$ say, is on the same arc as $\Delta_{1} \cap t$, and $D_{1} \cup \Delta$ separates off from $A$ a ball $B$ with $B \cap t$ a single unknotted arc. Sliding $D_{1}$ across $B$ reduces the intersection of $D$ and $\Delta$. The only remaining possible intersections are those simple closed curves that encircle both points of $\Delta \cap t$.

Note. It is a fairly simple exercise to construct examples to show that these final intersections cannot be removed by an isotopy fixing $t$ setwise.

4. An unsolved problem. As shown previously, any sum of prime tangles is prime, and any knotted sum of two untangles is a (2-bridge) prime knot. Is an arbitrary sum of a prime tangle with the untangle a prime knot or link? The answer is 'no' because any knot may be expressed in that form (see [KL] and the proof of Theorem 4). The way in which a tangle $(A, t)$ is added to the untangle can be specified by describing the simple closed curve in $\partial A-\partial t$ that identifies with the boundary of a disc separating the arcs of the untangle. Two ways of adding $(A, t)$ 
to the untangle will be regarded as the same if they correspond to isotopic simple closed curves in $\partial A-\partial t$.

Conjecture. Let $(A, t)$ be a prime tangle. Then there is at most one way of adding $(A, t)$ to the untangle to obtain a nonprime knot.

It has been pointed out by $\mathrm{F}$. Gonzales-Acuna that the conjecture is true for a prime algebraic tangle $(A, t)$, namely a partial sum of some untangles (e.g. tangles (a), (c), (d) and (e) of Figure 2). In outline this is because the double branched cover of such a tangle is the union of Seifert fibred manifolds with boundary, glued together over incompressible parts of their boundaries. The double branched cover of the untangle is a solid torus and there is just one way of gluing a solid torus to a boundary component of a Seifert fibre space that prevents the union being Seifert fibred. This corresponds to the way of adding on the untangle that must be avoided. Thus, except in this case, the resulting knot has a double branched cover that is a union of Seifert fibred pieces and hence is irreducible (one must check that $S^{1} \times S^{2}$ and $\mathbf{R} P^{3} \# \mathbf{R} P^{3}$ do not occur) and thus the knot is prime.

If the conjecture should be false it would be interesting to know if it be true with 'finitely many ways' in place of 'one way'.

Suppose a knot $\left(S^{3}, k\right)$ is formed by adding a prime tangle $(A, t)$ to an untangle, and $F$ is a 2 -sphere in $S^{3}$ meeting $k$ in two points. It is easy to isotop $F$ (fixing $k$ setwise) so that $F$ meets the untangle in a collection of parallel discs separating the arcs of the untangle; hence $F \cap A$ is a genus zero surface meeting $t$ in two points, and with the components of $\partial(F \cap A)$ parallel in $\partial A-\partial t$. A version of Proposition 6 and its corollary, generalized from discs to genus zero surfaces, would then solve the conjecture.

If the conjecture is true then knots with unknotting number equal to one are prime. This is another well-known problem [Gn]. A knot has unknotting number equal to one if it has a presentation in which the changing of one cross-over from an over-pass to an under-pass changes the knot to an unknot. Let $B$ be a small 3-ball 'enclosing a neighbourhood of such a cross-over' that meets the knot $k$ in an untangle. Let $(A, t)$ be the complementary tangle. Certainly $(A, t)$ contains no knotted arc-ball pairs since the untangle can be added to it to create the unknot. Thus $(A, t)$ is either untangled, in which case $k$ is prime as $b(k)=2$, or $(A, t)$ is prime in which case the primeness of $k$ would follow from the conjecture, for one way of adding the untangle gives the unknot (not prime) so any other way should give a prime knot.

Interpreted by means of double branched covers the conjecture asks if there is at most one way of gluing a solid torus to an irreducible, boundary-irreducible 3-manifold $M$ (with involution), $\partial M$ being a torus, to obtain a reducible 3-manifold. In the case of the problem concerning knots of knotting number one, the manifold is a knot complement. Then one is asking when surgery on a knot can produce a reducible 3-manifold not equal to $S^{3}$ (compare the property $R$ problem $[\mathbf{K}])$. It is known that $p q$-surgery on a $(p, q)$-cable knot gives a composite 3-manifold but this seems to be irrelevant to the problem. It has recently been shown by A. Hatcher and W. Thurston [HT], that any (Dehn) surgery on any 2-bridge knot 
that is not a torus knot yields an irreducible 3-manifold. Also, W. Menasco has the same result for all but finitely many Dehn surgeries on prime alternating (nontorus) knots.

The investigation of the prime tangle given in this paper is meant to add one more method to the established methods of proving primeness. These methods have already been mentioned; they are bridge number techniques, genus methods, double branched cover arguments and discussions of tori in the knot complement. For completeness it seems sensible to record that the last (torus) method has recently been extended by J. C. Gómez Larranaga [GL] to a theorem involving higher genus surfaces in the knot complement, and that W. Menasco [M] has a surprising algorithm for detecting composite knots from alternating presentations.

\section{REFERENCES}

[B] F. Bonahon (to appear).

[C] J. H. Conway, An enumeration of knots and links, and some of their algebraic properties, Computational Problems in Abstract Algebra, Pergamon Press, Oxford and New York, 1969, pp. 329-358.

[GL] J. C. Gómez Larrańaga, Knot primeness, Doctoral Dissertation, Cambridge University, 1981.

[Gk] R. E. Goodrick, Non-simplicially collapsible triangluations on $I^{n}$, Proc. Cambridge Philos. Soc. 64 (1968), 31-36.

[Gn] C. McA. Gordon, Problems in knot theory, Knot Theory Proceedings (Plans-sur-Bex, 1977), Lecture Notes in Math., vol. 685, Springer-Verlag, Berlin and New York, 1978, pp. 309-311.

[HT] A. Hatcher and W. Thurston, Incompressible surfaces in 2-bridge knot complements (to appear).

[Jo] W. Jaco, Lectures on three-manifold topology, CBMS Regional Conf. Ser. in Math., Number 43, Amer. Math. Soc., Providence, R. I., 1980.

[Jn] K. Johannson, Homotopy equivalences of 3-manifolds with boundaries, Lecture Notes in Math., vol. 761, Springer-Verlag, Berlin and New York, 1979.

[KT] P. K. Kim and J. L. Tollefson, Splitting the P.L. involutions of nonprime 3-manifolds, Michigan Math. J. 27 (1980), 259-274.

[K] R. Kirby, Problems in low dimensional manifold theory, Proc. Sympos. Pure Math., vol. 32, Amer. Math. Soc., Providence, R. I., 1978, pp. 273-312.

[KL] R. C. Kirby and W. B. R. Lickorish, Prime knots and concordance, Math. Proc. Cambridge Philos. Soc. 86 (1979), 437-441.

[L] C. Livingston, Homology cobordisms of 3-manifolds, knot concordances, and prime knots, Pacific J. Math. (to appear).

[M] W. Menasco, Incompressible surfaces in the complement of alternating knots and links (to appear).

[P1] K. A. Perko, A weak 2-bridged knot with at most three bridges is prime, Notices Amer. Math. Soc. 26 (1978), A-648 (and a preprint).

[P2] __ Invariants of eleven-crossing knots (to appear).

[S] H. Schubert, Uber eine numerische Knoteninvariante, Math. Z. 61 (1954), 245-288.

[W] F. Waldhausen, Über Involutionen der 3-Sphäre, Topology 8 (1969), 81-91.

Department of Pure Mathematics, University of Cambridge, 16 Mill Lane, Cambridge, CB2 ISB, ENGLAND 